Case Report

\title{
Efficacy of Autologous Stem Cell Transplantation for the Treatment of Multiple Myeloma in HIV-Positive Patients
}

\author{
Bin Ni, Nidhi Sharma *, Ashley Rosko, Don M. Benson Jr., Yvonne A. Efebera
}

Department of Internal Medicine, Division of Hematology, The Ohio State University Comprehensive Cancer Center, Columbus, Ohio, USA; E-Mails: Bin.Ni@vumc.org; Nidhi.Sharma@osumc.edu; $\quad$ Ashley.Rosko@osumc.edu; Yvonne.Efebera@ohiohealth.com

* Correspondence: Nidhi Sharma; E-Mail: Nidhi.Sharma@osumc.edu

Academic Editor: Alessandra Picardi

Special Issue: Autologous Stem Cell Transplantation

OBM Transplantation

2021, volume 5 , issue 3

doi:10.21926/obm.transplant.2103148
Received: April 12, 2021

Accepted: June 25, 2021

Published: July 09, 2021

\begin{abstract}
Prior to the advent of anti-retroviral therapy (ART), autologous stem cell transplant (ASCT) was relatively contraindicated for multiple myeloma (MM) patients with human immunodeficiency virus (HIV) due to issues associated with stem cell harvest and the risk of opportunistic infections. With the widespread use of ART for control of HIV, ASCT is now the preferred treatment for relapsed lymphoma, the leading hematopoietic malignancy associated with HIV/AIDS. Hence, MM patients with HIV controlled by ART may benefit equally from aggressive combination treatment of chemotherapy and ASCT. This study seeks to evaluate the clinical course and treatment outcomes of patients with HIV and MM treated with ASCT. Five male patients with average age 53.2 years were included in this study. Patients were diagnosed with HIV prior to diagnosis of MM and were appropriately treated with ART prior to ASCT. All patients had undetectable HIV viral load (VL) prior to ASCT, and remained undetectable after ASCT. Adequate CD34+ stem cells were collected. Patients received high dose melphalan $\left(200 \mathrm{mg} / \mathrm{m}^{2}\right)$ followed by ASCT. ART was continued during ASCT. Patients experienced usual ASCT toxicities including diarrhea, mucositis, and neutropenic fever. All
\end{abstract}

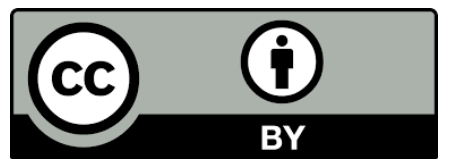

(c) 2021 by the author. This is an open access article distributed under the conditions of the Creative Commons by Attribution License, which permits unrestricted use, distribution, and reproduction in any medium or format, provided the original work is correctly cited. 
patients had normal neutrophil and platelet engraftments. $60 \%$ of patients had very good partial response or better after ASCT. All patients received post ASCT maintenance until progression/toxicity/patient decision. As of December 2020, 2 patients have died 51 and 85 months from ASCT due to other causes. The 3 remaining patients are alive 5-7 years after ASCT. MM patients with concurrent HIV infection that is controlled on ART would benefit from aggressive treatment with chemotherapy and ASCT, with continued ART as they tolerate ASCT as well as myeloma patients without HIV infection.

\section{Keywords}

Multiple Myeloma; HIV; anti-retroviral therapy

\section{Introduction}

Multiple myeloma (MM) represents approximately $10 \%$ of all hematologic malignancies [1] with over 20,000 new diagnoses and greater than 10,000 deaths in the United States per year [2]. MM is characterized by uncontrolled proliferation of plasma cell producing monoclonal immunoglobulins.

The severe immunodeficiency caused by advanced HIV infection has been recognized as capable of contributing to different types of cancers [3]. Persons living with HIV (PLWH) suffer from higher rates of malignancies, especially those of the B-cell lineage, which includes plasma cell disorders [46]. While lymphoma is the leading hematopoietic malignancy in PLWH higher rates of MM and worse outcomes may be observed among HIV-infected individuals [7]. There is a paucity of expertise in the treatment of individuals with these co-morbid conditions [8]. The introduction of high dose therapy (HDT) and autologous stem cell transplant (ASCT) in the 1980s was a major advancement in MM treatment. Prior to the advent of anti-retroviral therapy (ART), ASCT was relatively contraindicated for patients with HIV and hematological malignancies due to issues associated with stem cell harvest and the risk of opportunistic infections. With the widespread use of ART for HIV control, high dose chemotherapy and ASCT is now the preferred treatment for relapsed lymphoma among PLWH [9]. It stands to reason that MM patients with HIV controlled by ART may benefit equally from aggressive combination treatment of chemotherapy and ASCT. Very little published data is available examining outcomes of ASCT in patients with HIV and MM $[4,10,11]$. This report will evaluate the clinical course and outcomes of PLWH with MM treated with high dose therapy and ASCT at a single center.

\section{Methods}

Between August 2002 and June 2016, 5 patients who met the diagnostic criteria for symptomatic MM and were HIV positive underwent HDT chemotherapy and ASCT at The Ohio State University Medical Center. Standard criteria for ASCT eligibility were met. All patients received conditioning regimen with melphalan $200 \mathrm{mg} / \mathrm{m}^{2}$. Filgrastim $5 \mathrm{ug} / \mathrm{kg}$ was administered subcutaneously daily from day 1 after ASCT until recovery of absolute neutrophil count (ANC) to >1.5 $\times 10^{9} / \mathrm{L}$ for 3 days. Patients received infection prophylaxis with antiviral (acyclovir) and antifungal (fluconazole) prophylaxis. All patients remained on pre-ASCT ART regimen throughout stem cell collection and 
OBM Transplantation 2021; 5(3), doi:10.21926/obm.transplant.2103148

ASCT (Table 1). All patients were started on post ASCT maintenance until disease progression, toxicities or patient decision to stop. 
Table 1 Patient Characteristics.

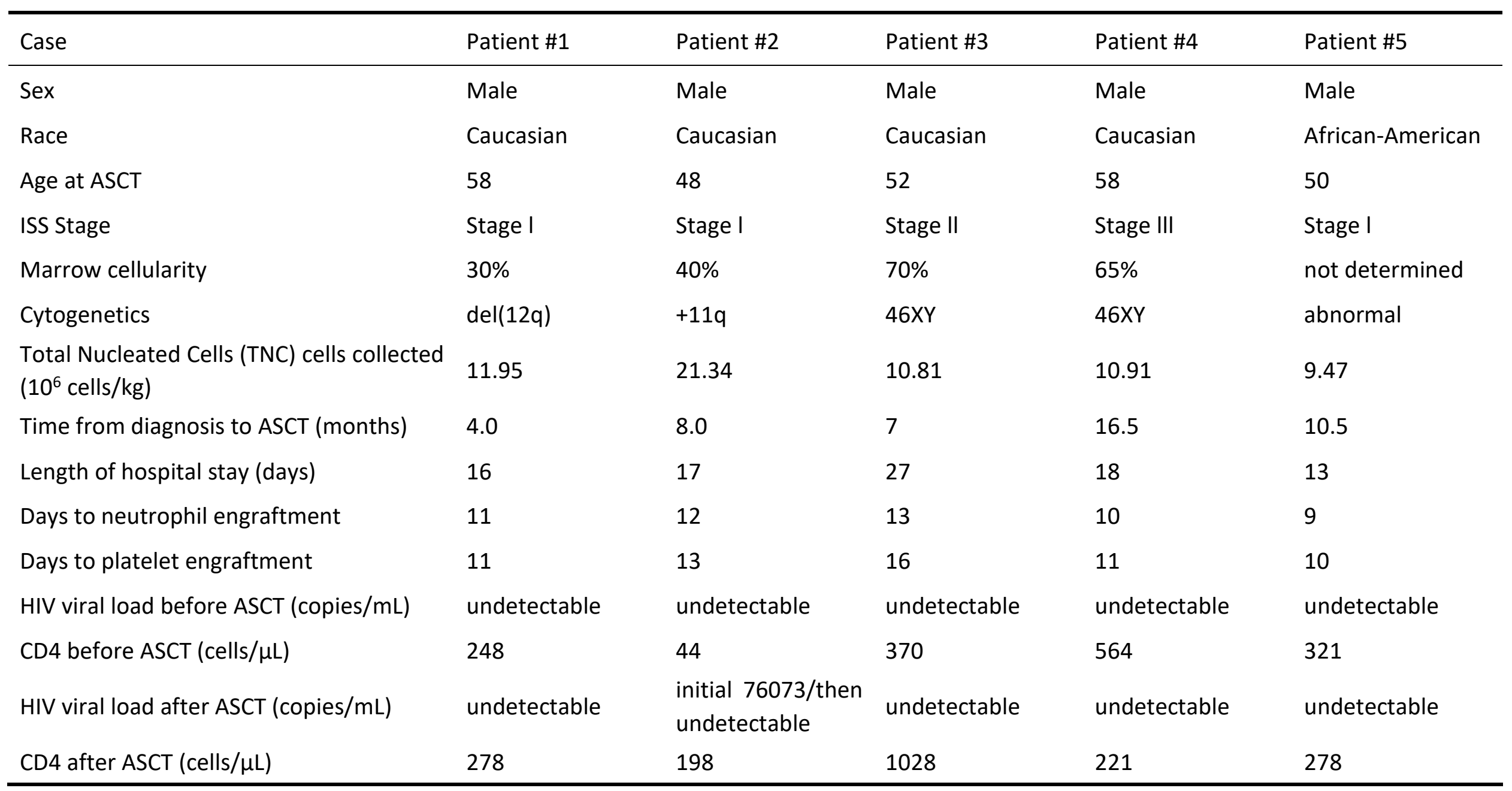




\section{Case Summaries}

\subsection{Case 1}

A 58-year-old Caucasian male presented to the emergency department in 2013 with several months of progressive back pain. Past medical history was significant for HIV on ART (diagnosed in 1995) and hepatitis B. Imaging showed an anterior chest wall mass as well as multiple lytic lesions throughout the spine. CT guided core biopsy of the chest wall mass was consistent with a lambda light chain plasmacytoma. At diagnosis, bone marrow plasmacytosis was 30\%, hemoglobin $10.6 \mathrm{mg} / \mathrm{dl}$ ( $\mathrm{nl} 13.2-16.5$ ), creatinine $1.6 \mathrm{mg} / \mathrm{dl}$ ( $\mathrm{nl} 0.7-1.3$ ), 24-hr urine lambda light chain $8.9 \mathrm{~g} /$ day, calcium $9.5 \mathrm{mg} / \mathrm{dl}$ ( $\mathrm{nl}$ 8.6-10.5), free lambda chain $5350 \mathrm{mg} / \mathrm{l}$ ( $\mathrm{nl}$ 5.6-26.3), and $\beta-2$ microglobulin $3.0 \mathrm{mg} / \mathrm{L}(\mathrm{nl}<2.11)$. He was diagnosed with lambda light chain MM stage I by International Staging System (ISS) criteria and was standard risk according to FISH with $13 q$ deletion. He initiated treatment with standard dose VRD (bortezomib, lenalidomide, dexamethasone) and radiation of the chest wall mass and bilateral ribs with total $20 \mathrm{~Gy}$. He achieved a stringent complete (CR) remission after 4 cycles of VRD. He underwent collection of $7.71 \times 10^{6}$ cells $/ \mathrm{kg}$ CD34+ stem cells and went on to ASCT 4 months after initial diagnosis. HIV viral load (VL) was undetectable with CD4+ cell count 321 cells/ $\mu \mathrm{L}$ prior to ASCT. ASCT course was complicated by culture-negative febrile neutropenia managed with empiric antibiotics. ART was maintained throughout stem cell mobilization and ASCT. Patient achieved both neutrophil and platelet engraftment on day 11 posttransplant. HIV VL remained undetectable with CD4+ cell count 278 cells/ $\mu \mathrm{L}$ after ASCT. He initiated lenalidomide maintenance but progressed 53 months post-ASCT. He subsequently received pomalidomide/ixazomib/dexamethasone followed by a second ASCT with melphalan conditioning. He then obtained a CR and continues maintenance therapy with pomalidomide and ixazomib with no evidence of progression (Table 2). 
Table 2 Response and Survival Post ASCT.

\begin{tabular}{|c|c|c|c|c|c|c|c|}
\hline Patient & $\begin{array}{l}\text { Disease status } \\
\text { post ASCT }\end{array}$ & $\begin{array}{l}\text { Disease status } \\
\text { with } \\
\text { Maintenance }\end{array}$ & $\begin{array}{l}\text { Progression } \\
\mathrm{Y} / \mathrm{N}\end{array}$ & $\begin{array}{l}\text { Time to } \\
\text { Progression } \\
\text { (mos) }\end{array}$ & Death $\mathrm{Y} / \mathrm{N}$ & $\begin{array}{l}\text { Time from ASCT to } \\
\text { death/last follow- } \\
\text { up (mos) }\end{array}$ & Cause of Death \\
\hline 1 & sCR & SCR & $Y$ & 53 & $\mathrm{~N}$ & 89 & \\
\hline 2 & SCR & SCR & $\mathrm{N}$ & & $Y$ & 85 & EBV Lymphoma \\
\hline 3 & PR & PR & $\mathrm{N}$ & & $Y$ & 51 & AML \\
\hline 4 & VGPR & $\mathrm{CR}$ & $Y$ & 39 & $\mathrm{~N}$ & 55 & \\
\hline 5 & MR & PR & $\mathrm{N}$ & & $\mathrm{N}$ & 53 & \\
\hline
\end{tabular}

CR, complete response; sCR stringent CR; EBV Epstein Barr Virus; PR partial response; AML acute myelogenous leukemia; VGPR very good partial response. 


\subsection{Case 2}

A 48-year-old Caucasian male presented to the emergency department in summer 2012 with complaint of left rib pain. Past medical history included HIV on ART (diagnosed in 1997), immune thrombocytopenia (ITP), and hepatitis B. He was initially treated symptomatically but underwent plain radiographs and MRI after several months of persistent and progressive symptoms. Imaging demonstrated a T11 burst fracture as well as lesions to the left iliac and left fourth rib. He subsequently underwent orthopedic intervention with spinal fusion and vertebral body biopsy; pathology demonstrated a kappa restricted plasma cell neoplasm. He had further workup including bone marrow biopsy that showed normal cellularity with $<5 \%$ plasma cells and normal FISH. Skeletal survey had multiple lytic lesions. He was diagnosed with IgG kappa MM ISS Stage I, standard risk. He initiated treatment with reduced dose RD (lenalidomide, dexamethasone) given underlying chronic stable ITP along with radiation to the T11 lesion with total $25 \mathrm{~Gy}$. He achieved a stringent $\mathrm{CR}$ after 6 cycles of RD. $6.24 \times 10^{6}$ cells/kg CD34+ stem cells were collected from peripheral blood followed by ASCT approximately 8 months after initial diagnosis. HIV VL was undetectable with CD4+ cell count 44 cells/ $\mu \mathrm{L}$ prior to ASCT. ASCT course was complicated by culture negative febrile neutropenia treated with empiric antibiotics and non-infectious diarrhea. ART was continued throughout stem cell mobilization and ASCT. Patient achieved neutrophil engraftment on day 12 and platelet engraftment on day 13 post-transplant. He experienced HIV viral rebound shortly after ASCT, but HIV VL was subsequently suppressed without change in ART and CD4+ cell count improved to 198 cells $/ \mu \mathrm{L}$ after ASCT. He initiated lenalidomide maintenance therapy with weekly dexamethasone (for ITP) after ASCT. Unfortunately, he developed EBV-associated large cell lymphoma refractory to treatment and died 85 months from ASCT but had no evidence of myeloma progression at time of death (Table 2).

\subsection{Case 3}

A 52-year old Caucasian male presented to the emergency department in 2015 with complaint of urinary symptoms along with fever and malaise. Past medical history was significant for HIV on ART, diagnosed $\geq 5$ years prior to time of presentation, hepatitis B, type 2 diabetes mellitus, and benign prostatic hyperplasia with recurrent urinary tract infections. He was admitted for sepsis secondary to urinary tract infection and was noted to have new renal insufficiency with nephropathy, which prompted further workup that resulted in abnormal serum protein electrophoresis showing presence of IgG kappa monoclonal protein $(1954 \mathrm{mg} / \mathrm{dl}$ ) with elevated kappa light chains (462 mg/l). Bone marrow biopsy with 15\% atypical kappa positive plasma cells. Skeletal survey and MRI of spine demonstrated multiple subcentimeter lesions in the thoracic and sacral vertebral bodies. He was diagnosed with IgG kappa MM stage II standard risk. He underwent treatment with VRD with partial response (PR) after 5 cycles. $4.11 \times 10^{6}$ cells $/ \mathrm{kg}$ CD34+ stem cells were collected from peripheral blood and he proceeded to ASCT approximately 7 months after initial diagnosis. HIV VL was undetectable with CD4+ cell count 370 cells/ $\mu \mathrm{L}$ prior to ASCT. ASCT course was complicated by stay in intensive care unit for septic shock requiring vasopressors, atrial fibrillation with rapid ventricular response, and small bowel obstruction (SBO). Septic shock was treated with empiric broad-spectrum antibiotics, he was placed on diltiazem for heart rate control, and SBO was managed non-surgically but did require period of total parenteral nutrition. ART was continued during stem cell mobilization and ASCT. Patient achieved neutrophil engraftment on day 
13 and platelet engraftment on day 16 post-transplant. HIV VL remained undetectable with CD4+ 1028 cells/ $\mu \mathrm{L}$ after ASCT. He initiated lenalidomide maintenance therapy post-ASCT. Unfortunately, he developed a second primary malignancy of acute myelogenous leukemia 49 months from ASCT and died 2 months later (Table 2).

\subsection{Case 4}

A 58-year old Caucasian male presented to his primary care physician in 2015 with complaint of 2 months of progressive pelvic edema followed by urinary and fecal urgency/frequency. Past medical history was significant for HIV on ART, diagnosed in 1992. He underwent MRI of the pelvis for evaluation and was found to have a large pelvic mass with significant invasion of local structures. He was subsequently admitted for further evaluation. ACT-guided core biopsy of the pelvic mass was consistent with a plasmacytoma. Serum protein electrophoresis showed presence of IgG kappa monoclonal protein $(3915 \mathrm{mg} / \mathrm{dL})$ with elevated kappa free light chains $(1172.5 \mathrm{mg} / \mathrm{l})$ and $\beta-2$ microglobulin $7.8 \mathrm{mg} / \mathrm{L}$. He underwent a bone marrow biopsy demonstrating hypercellular bone marrow (65\%) with 3\% plasma cells; karyotype was normal. Other labs included hemoglobin 13.7 $\mathrm{mg} / \mathrm{dL}$, creatinine $1.11 \mathrm{mg} / \mathrm{dL}$, and calcium $8.4 \mathrm{mg} / \mathrm{dL}$. He was diagnosed with IgG Kappa MM ISS Stage III, standard risk. He underwent induction therapy with CyBorD (cyclophosphamide, bortezomib, dexamethasone) with stable disease after 7 cycles. During therapy, he developed a left lower extremity DVT and was treated with lovenox. Plans were made to proceed to ASCT but premobilization workup showed evidence of disease progression while off therapy. Consequently, ASCT was deferred and patient was initiated on second line treatment with VRD. He achieved very good PR after 4 cycles of VRD. $5.56 \times 10^{6}$ cells $/ \mathrm{kg}$ CD34+ cells were collected from peripheral blood and he proceeded to ASCT approximately 16 months after initial diagnosis. HIV VL was undetectable with CD4+ cell count 564 cells/ $\mu \mathrm{L}$ prior to ASCT. ASCT course was complicated by non-infectious diarrhea, and drug-induced esophagitis that was treated symptomatically. ART was continued during stem cell mobilization and ASCT. Patient achieved neutrophil engraftment on day 10 and platelet engraftment on day 11 post-transplant. HIV viral load remained undetectable with CD4+ 221 cells $/ \mu \mathrm{L}$ after ASCT. He received 2 years of bortezomib maintenance therapy following ASCT, obtaining a CR, but then decided to stop. His disease progressed 39 months following ASCT. He continues on salvage therapy (Table 2 ).

\subsection{Case 5}

A 50-year old African-American male with past medical history significant for HIV on ART, (diagnosed in 2001), hepatitis B, history of myocardial infarction. Proteinuria was found on routine labs and was referred to nephrology in 2015 by his infectious disease provider. Serum protein electrophoresis detected presence of an $\mathrm{M}$ protein spike and was referred to an oncologist for further evaluation. He underwent bone marrow biopsy that demonstrated $70 \%$ lambda positive plasma cells. Other labs included $M$ protein $1751 \mathrm{mg} / \mathrm{dl}$, lambda light chain $99.5 \mathrm{mg} / \mathrm{l}, \beta-2$ microglobulin $2.5 \mathrm{mg} / \mathrm{L}$, normal hemoglobin, creatinine, and calcium levels. Skeletal survey demonstrated a large lytic lesion in the L2 vertebral body. He was diagnosed with IgG lambda MM ISS Stage I, standard risk. He initiated induction therapy with VRD and completed 6 cycles with minimal response (MR); he also developed an $\mathrm{L} 2$ compression fracture during treatment and underwent vertebroplasty prior to ASCT. CD34+ stem cells were collected from peripheral blood 
with a total $8.52 \times 10^{6}$ cells $/ \mathrm{kg}$ CD34+ cells retrieved. He went on to ASCT approximately 10 months after initial diagnosis. HIV VL was undetectable with CD4+ cell count 321 cells/ $\mu \mathrm{L}$ prior to ASCT. ASCT course was complicated by mucositis, non-infectious diarrhea, and asymptomatic hypotension; no additional anti-infectives beyond standard prophylaxis was given. ART was continued during stem cell mobilization and ASCT. Patient achieved neutrophil engraftment on day 9 and platelet engraftment on day 10 post-transplant. HIV viral load remained undetectable with CD4+ 278 cells/ $\mu \mathrm{L}$ after ASCT. He began lenalidomide maintenance therapy following ASCT and has achieved a PR (Table 2). At last, follow-up patient's myeloma disease has remained stable.

\section{Conclusions}

HIV infection is associated with an increased risk of hematologic malignancies compared with that in the general population $[12,13]$. Multiple myeloma is less commonly associated with HIV than non-Hodgkin lymphoma, but PLWH with HIV have been reported to have higher rates of monoclonal gammopathy [7, 8] along with an increased relative risk of developing MM over time after HIV diagnosis [14]. Several studies have shown that outcomes of chemotherapy and ASCT for treatment of lymphoma are similar among PLWH and patients without HIV infection $[15,16]$ however, experiences of ASCT for treatment of MM in PLWH are extremely limited and PLWH are generally excluded from MM stem cell transplant trials.

In the pre-ART era, few studies attempted to extend ASCT to PLWH because it was difficult to manage treatment-related complications for patients with HIV-related hematologic malignancies. Investigators from France reported ASCT in HIV-positive patients with lymphoma for the first time in 1996, however, the post-transplant course was accompanied by multiple opportunistic infections [17]. In the post-ART era, effective suppression of HIV replication and improved immune function have led to reductions in the incidences of opportunistic infections and HIV-related morbidity and mortality. As a result, numerous studies have shown that the outcomes in HIV-positive patients and HIV-negative patients with non-Hodgkin lymphoma after ASCT are similar [18, 19].

Our experiences of PLWH with myeloma have shown that when HIV is adequately controlled with ART, clinical outcomes following chemotherapy and ASCT similar to patients without HIV infection. All patients in this report tolerated induction therapy and underwent autologous stem cell mobilization and adequate CD34+ cells collection (median $7 \times 10^{6}$ cells $/ \mathrm{kg}$ ) without issues. All patients in this report received high-dose melphalan conditioning and were able to continue their usual ART regimen throughout the transplant period. All patients started on maintenance and continued until disease progression. During ASCT it is important to monitor closely for drug toxicities due to potential drug-drug interactions between antiretroviral agents and drugs used in myeloablative regimens. Neutrophil engraftment time for these patients (median 11 days) was similar to that in patients without HIV infection $[20,21]$. Responses to therapy and overall survival are also similar to non-HIV patients $[20,21]$. Our report highlights the therapeutic benefit of aggressive MM therapy in patients with well-controlled HIV infection and demonstrates the feasibility of high dose therapy and ASCT with maintenance of ART during transplant for HIVinfected patients. 


\section{Authorship Contributions}

Conception and design: YE; collection and assembly of data: BN, YE; manuscript writing: NS, BN, YE; scientific input and critical comments: AR, DB.

\section{Conflict of Interest Disclosures}

The authors declare no competing financial interests.

\section{References}

1. Rajkumar SV. Multiple myeloma: Every year a new standard? Hematol Oncol. 2019; 37: 62-65.

2. Siegel RL, Miller KD, Jemal A. Cancer Statistics. CA A Cancer J Clin. 2018; 68: 7-30.

3. Yee TT, Murphy K, Johnson M, Abdalla SH, Patton GS, Lee CA, et al. Multiple myeloma and human immunodeficiency virus-1 (HIV-1) infection. Am J Hematol. 2001; 66: 123-125.

4. Muzaffar J, Usmani S, Abdallah AO, Anaissie E, Cottler-Fox M, Restrepo A. High-dose chemotherapy and autologous stem cell transplantation for multiple myeloma in HIV-positive patients in the highly active antiretroviral therapy era: The myeloma institute of research and therapy experience. Clin Lymphoma Myeloma Leuk. 2013; 13: 171-174.

5. Rabkin CS. Association of non-acquired immunodeficiency syndrome-defining cancers with human immunodeficiency virus infection. J Natl Cancer Inst Monogr. 1998; 1998: 23-25.

6. Goedert JJ, Coté TR, Virgo P, Scoppa SM, Kingma DW, Gail MH, et al. Spectrum of AIDSassociated malignant disorders. Lancet. 1998; 351: 1833-1839.

7. Bandera A, Colella E, Clerici M, Rizzardini G, Gori A. The contribution of immune activation and accelerated aging in multiple myeloma occurring in HIV-infected population. Aids. 2018; 32: 2841-2846.

8. Fiorino AS, Atac B. Paraproteinemia, plasmacytoma, myeloma and HIV infection. Leukemia. 1997; 11: 2150-2156.

9. Balsalobre P, Díez-Martín JL, Re A, Michieli M, Ribera JM, Canals C, et al. Autologous stem-cell transplantation in patients with HIV-related lymphoma. J Clin Oncol. 2009; 27: 2192-2198.

10. Ambinder RF, Capoferri AA, Durand CM. Haemopoietic cell transplantation in patients living with HIV. Lancet HIV. 2020; 7: e652-e660.

11. Johnston C, Harrington R, Jain R, Schiffer J, Kiem HP, Woolfrey A. Safety and efficacy of combination antiretroviral therapy in human immunodeficiency virus-infected adults undergoing autologous or allogeneic hematopoietic cell transplantation for hematologic malignancies. Biol Blood Marrow Transplant. 2016; 22: 149-156.

12. Engels EA, Biggar RJ, Hall HI, Cross $\mathrm{H}$, Crutchfield A, Finch JL, et al. Cancer risk in people infected with human immunodeficiency virus in the United States. Int J Cancer. 2008; 123: 187-194.

13. Frisch M, Biggar RJ, Engels EA, Goedert JJ. Association of cancer with AIDS-related immunosuppression in adults. Jama. 2001; 285: 1736-1745.

14. Grulich AE, Li Y, McDonald A, Correll PK, Law MG, Kaldor JM. Rates of non-AIDS-defining cancers in people with HIV infection before and after AIDS diagnosis. Aids. 2002; 16: 1155-1161.

15. Díez-Martín JL, Balsalobre $P, \operatorname{Re} A$, Michieli M, Ribera JM, Canals $C$, et al. Comparable survival between HIV+ and HIV- non-Hodgkin and Hodgkin lymphoma patients undergoing autologous peripheral blood stem cell transplantation. Blood. 2009; 113: 6011-6014. 
16. Krishnan A, Palmer JM, Zaia JA, Tsai NC, Alvarnas J, Forman SJ. HIV status does not affect the outcome of autologous stem cell transplantation (ASCT) for non-Hodgkin lymphoma (NHL). Biol Blood Marrow Transplant. 2010; 16: 1302-1308.

17. Gabarre J, Leblond V, Sutton L, Azar N, Jouan M, Boccaccio C, et al. Autologous bone marrow transplantation in relapsed HIV-related non-Hodgkin's lymphoma. Bone Marrow Transplant. 1996; 18: 1195-1197.

18. Krishnan A, Molina A, Zaia J, Smith D, Vasquez D, Kogut N, et al. Durable remissions with autologous stem cell transplantation for high-risk HIV-associated lymphomas. Blood. 2005; 105: 874-878.

19. Re A, Michieli M, Casari S, Allione B, Cattaneo C, Rupolo M, et al. High-dose therapy and autologous peripheral blood stem cell transplantation as salvage treatment for AIDS-related lymphoma: Long-term results of the Italian Cooperative Group on AIDS and Tumors (GICAT) study with analysis of prognostic factors. Blood. 2009; 114: 1306-1313.

20. McCarthy PL, Owzar K, Hofmeister CC, Hurd DD, Hassoun H, Richardson PG, et al. Lenalidomide after stem-cell transplantation for multiple myeloma. N Engl J Med. 2012; 366: 1770-1781.

21. Attal M, Lauwers-Cances V, Marit G, Caillot D, Moreau P, Facon T, et al. Lenalidomide maintenance after stem-cell transplantation for multiple myeloma. N Engl J Med. 2012; 366: 1782-1791.

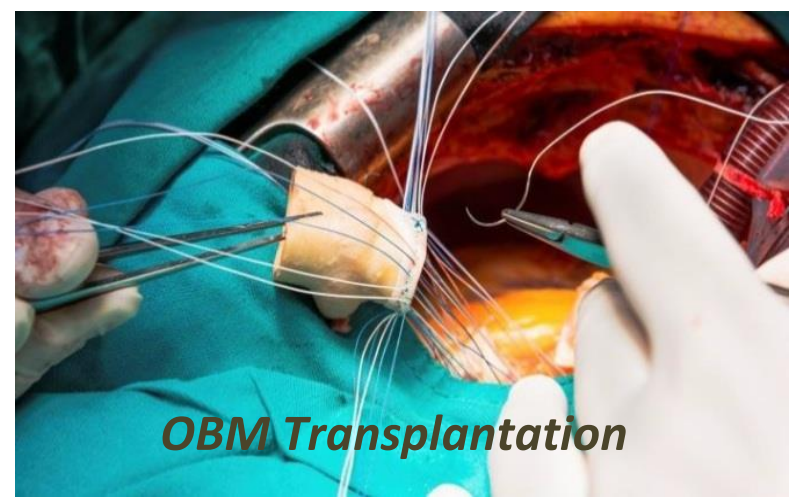

Enjoy OBM Transplantation by:

1. Submitting a manuscript

2. Joining in volunteer reviewer bank

3. Joining Editorial Board

4. Guest editing a special issue

For more details, please visit:

http://www.lidsen.com/journals/transplantation 